\title{
Near-field radio-frequency imaging by spin-locking with a nitrogen-vacancy spin
} sensor

\author{
Shintaro Nomura, ${ }^{1, a)}$ Koki Kaida, ${ }^{1}$ Hideyuki Watanabe, ${ }^{2}$ and Satoshi Kashiwaya ${ }^{3}$
}

1) Division of Physics, University of Tsukuba, Tennodai, Tsukuba, Ibaraki, 305-8571, Japan

${ }^{2)}$ National Institute of Advanced Industrial Science and Technology (AIST), Central2, Umezono, Tsukuba, Ibaraki, 305-8568, Japan

3) Department of Applied Physics, Nagoya University, Chikusa-Ku, Nagoya, 464-8571, Japan

(Dated: 1 June 2021)

We present results of near-field radio-frequency $(\mathrm{RF})$ imaging at micrometer resolution using an ensemble of nitrogen-vacancy (NV) centers in diamond. The spatial resolution of RF imaging is set by the resolution of an optical microscope, which is markedly higher than the existing RF imaging methods. High sensitivity RF field detection is demonstrated through spin locking. SCROFULOUS composite pulse sequence is used for manipulation of the spins in the NV centers for reduced sensitivity to possible microwave pulse amplitude error in the field of view. We present procedures for acquiring an RF field image under spatially inhomogeneous microwave field distribution, and demonstrate a near-field RF imaging of an RF field emitted from a photolithographically defined metal wire. The obtained RF field image indicates that the RF field intensity has maxima in the vicinity of the edges of the wire, in accord with a calculated result by a finite-difference time-domain method. Our method is expected to be applied in a broad variety of application areas, such as material characterizations, characterization of RF devices, and medical fields.

a)nomura.shintaro.ge@u.tsukuba.ac.jp 


\section{INTRODUCTION}

Electromagnetic field plays an increasingly important role for nondestructive imaging at $\mathrm{MHz}$ to sub $\mathrm{THz}$ frequency range. Near-field as well as far-field electromagnetic field imaging techniques are widely used in nondestructive imaging applications such as clinical applications, testing of infrastructure, and material characterization ${ }^{1,2}$. Whereas the near-field microwave imaging has already been widely used, the imaging at the $\mu \mathrm{m}$ scale resolution has been demonstrated only recently. By the introduction of a quantum sensing technique utilizing nitrogen-vacancy (NV) centers in diamond, microwave imaging has been demonstrated in micrometer resolution by mapping Rabi oscillation frequency driven by the microwave field ${ }^{3}$. The Rabi oscillation was detected by the photoluminescence (PL) from the negatively charged $\mathrm{NV}\left(\mathrm{NV}^{-}\right)$centers that reflects the spin states of the electrons in the NV centers. The emission probability of the optically excited state of the $|0\rangle$ state is larger than that of the $|1\rangle$ state because the $|1\rangle$ state has a decay channel via a metastable dark state. ${ }^{4}$ Then, the spin state is detected by the optical contrast between the $|0\rangle$ and $|1\rangle$ states. Due to the high density of the NVs, the spatial resolution of the electromagnetic field imaging is only set by the resolution of the optical system for collecting the PL. The frequency of the microwave to be detected was selected by tuning the resonance frequency of NV centers by the external magnetic field. The frequency range was practically limited to be around $1 \mathrm{GHz}$ at the lower bound due to the magnitude of the magnetic field available by a permanent magnet ${ }^{3}$, but may be extended above $100 \mathrm{GHz}$ by using a superconducting magnet $^{5}$.

Extension of the lower frequency bound of the electromagnetic field to be imaged may find broad application fields such as in material characterizations, characterization of RF devices, and medical fields. Most of the existing technology for electromagnetic field imaging utilizes sensor arrays with electric wiring. As a result, their spatial resolution and the number of sensing elements are limited by the sensor size and the number of wires. ${ }^{6-8}$ The electromagnetic field imaging using NV centers in diamond is free from this limitation because acquisition of an image is performed all optically by collecting the PL from NV centers. Furthermore, quantum sensing techniques enable high precision and sensitivity of electromagnetic field detection.

Here the lower bound of the frequency range of near-field electromagnetic imaging is ex- 
tended to $\mathrm{MHz}$ range by adopting the spin-locking technique. The spin-locking technique was originally developed for $\mathrm{NMR}^{9,10}$, and was later applied to atomic vapours ${ }^{11,12}$, a superconducting qubit ${ }^{13}$, and a single NV center ${ }^{14,15}$. In the spin-locking technique applied to NV centers, after initializing the spin state to the $|0\rangle$ state by a laser pulse, superposition of $|0\rangle$ and $|1\rangle$ states is prepared by a $\pi / 2$ microwave pulse to rotate the spin state about the $x$ axis in the rotating frame. Then a $y$-driving microwave field $\Omega_{\mathrm{d}} \cos \left(\omega_{0} t\right) \sigma_{y}$, where $\Omega_{\mathrm{d}}$ and $\omega_{0}$ are a Rabi frequency and a microwave frequency of the $y$-driving microwave field, respectively, is applied in the direction parallel to the spin in the rotating frame. This creates an energy gap $\Omega_{\mathrm{d}}$ between the dressed states $|+\rangle=\frac{1}{\sqrt{2}}(|0\rangle+i|1\rangle)$ and $|-\rangle=\frac{1}{\sqrt{2}}(|0\rangle-i|1\rangle)$. This energy gap $\Omega_{\mathrm{d}}$ protects the electron spin from the broad external noise off-resonant to $\Omega_{\mathrm{d}}$. Alternatively, the dressed states enable highly sensitive detection of RF signal on resonant to $\Omega_{\mathrm{d}}$. This ensures high accuracy and high sensitivity of the electromagnetic field imaging based on the spin-locking as compared to the method based on the Rabi oscillation ${ }^{3}$.

The amplitude of the microwave field may not be homogeneous in the field-of-view of wide-field microscopy. This may lead to pulse length or amplitude errors in the microwave pulses and may degrade the fidelity of the spin manipulation. Composite pulse sequences have been developed to performs desired rotation with reduced sensitivity to systematic errors in the pulse such as frequency and amplitude ${ }^{16-19}$. Degradation of the near-field $\mathrm{RF}$ image by possible microwave pulse amplitude error is expected to be much reduced by adopting a composite pulse sequence to the spin-locking technique.

In this paper, we describe near-field RF imaging using a spin-locking technique at micrometer spatial resolution using an ensemble of NV centers in diamond. Methods for near-field RF imaging based on wide-field microscopy and for simulation of RF field are described in Sec. II. The rotating-frame relaxation time $T_{1 \rho}$ is examined in Sec. III A and the noise spectrum is presented in Sec. III B. Sensitivity to possible microwave amplitude error is examined in Sec. III C and the RF image is shown in Sec. III D. Finally conclusions are given in Sec. IV.

\section{METHODS}

Figures 1(a)-1(c) show pulse sequences for spin-locking measurement. After initialization to the $|0\rangle$ state by a laser pulse from a temperature-stabilized pulsed laser diode operating 
at $520 \mathrm{~nm}$ (Figs. 1(a), 1(d)), a $\pi / 2$ microwave pulse is applied in a standard measurement scheme for a rotation about the $x$-axis in the rotating frame (Fig. 1(e)). The dressed states are prepared by applying the $y$-driving field with Rabi frequency $\Omega$ with a duration $\tau$ (Fig. $1(\mathrm{e}))$. By adopting a phase cycling scheme, the second $\pi / 2$ microwave pulse is applied with a positive (pattern A) (Fig. 1(f)) or a negative (pattern B) (Fig. 1(g)) sign to eliminate the common-mode noise and to enhance the detected signal magnitude. This sequence is repeated by $n$ cycles, during which period PL intensity images for pattern $\mathrm{A}\left(I_{A}\right)$ and $\mathrm{B}\left(I_{B}\right)$ are acquired by a scientific CMOS camera. Then the images of $\left(I_{\mathrm{A}}-I_{\mathrm{B}}\right) / I_{\mathrm{B}}$ are calculated for each $\tau$. The obtained images reflect the population difference between the final spin states $|0\rangle$ and $|1\rangle$ (Figs. 1(f)-1(g)).

The rotation angle of the spin deviates from the nominal value of $\pi / 2$ if the amplitude of the microwave pulse varies from the calibrated value. Under this amplitude error, the spin after the first $\pi / 2$ pulse (Fig. 1(e)) does not direct to the equator of the Bloch sphere and deviates from the direction of the $y$-driving microwave pulse. The error in the manipulation of the spin is further accumulated by the second $\pi / 2$ pulse. As a result, the spin-locking signal becomes smaller than the ideal case using exact $\pi / 2$ pulses due to the error in the pulse amplitude.

The sensitivity to systematic errors in the pulse amplitude in the field-of-view of an RF image is reduced by using a composite pulse sequence. By applying multiple pulses with possibly different lengths and phases, the variable parameters of the pulses increase. The effect of the pulse errors from a target rotation angle is reduced by properly choosing the pulse parameters at the cost of the longer total pulse length. The increase in the total irradiation time of microwave pulses, however, may deteriorate the fidelity in a system with a finite spin dephasing time $T_{2}^{*}$. Hence, we choose SCROFULOUS composite pulse sequence (Figs. 1(b), 1(c)) that has an advantage in short total pulse length ${ }^{17,18}$ and is suitable for a system with short $T_{2}^{*}$, for manipulation of the spins in the NV centers. The SCROFULOUS pulse sequence is composed of three pulses

$$
\theta_{1} \phi_{1}-\theta_{2} \phi_{2}-\theta_{3} \phi_{3}
$$

where $\theta_{i}$ and $\phi_{i}$ are the nominal rotation angle and the phase angle, respectively. The time symmetric case of $\theta_{1}=\theta_{3}$ and $\phi_{1}=\phi_{3}$ is used, which ensures that the rotation is a rotation about an axis in the $x y$ plane. For a nominal $\pi / 2$ rotation about the $x$-axis, we 
have $\theta_{1}=115.2^{\circ}, \phi_{1}=62.0^{\circ}, \theta_{2}=180^{\circ}$, and $\phi_{2}=280.6^{\circ}$ (Fig. $1(\mathrm{c})$ ). Shaped pulses ${ }^{20}$ were used for reduction of the bandwidths of the baseband and for accurate control of the widths of the microwave pulses.

Microwave pulses were generated by up-converting the baseband I and Q pulses from an arbitrary wave generator (33622A, Keysight) by a double-balanced mixer (IQ-1545, Marki) and a local oscillator (SMC100A, Rhodes-Schwarz). After amplifying with an amplifier (ZHL-16W-43+, Mini-Circuits), a spatially homogeneous microwave field in the field of view of the microscope image was applied by a microwave planar ring antenna ${ }^{21}$ placed above a diamond chip (Fig. 1(h)). By ion-implantation ${ }^{3,22}$ of ${ }^{15} \mathrm{~N}_{2}^{+}, \mathrm{NV}$ centers were created at about $10 \mathrm{~nm}$ below the surface of a (100) CVD type IIa ultra-pure diamond chip with a size of $2.0 \times 2.0 \times 0.5 \mathrm{~mm}^{3}$, followed by annealing at $800^{\circ} \mathrm{C}$ and acid treatment. Inhomogeneous dephasing time $T_{2}^{*}$ and Hahn-Echo pure dephasing time $T_{2}$ were estimated to be 0.8 and 4 $\mu \mathrm{s}$, respectively. Photoluminescence from NV centers was imaged by a home-made widefield microscope equipped with a scientific CMOS camera (Zyla5.5, Andor) and an objective lens $100 \times$ with an NA 0.73 and a working distance of $4.7 \mathrm{~mm}$ at room temperature after passing through an optical long-pass filter with the cut-off wavelength of $650 \mathrm{~nm}$ (Fig. 1(h)). The pulse laser diode, arbitrary wave generator (33622A, Keysight), microwave switch, and the scientific CMOS camera were controlled by a pulse sequencer (Pulse Blaster ESR Pro, Spincore). A photolithography-defined metal wire was placed below the diamond chip. A continuous RF field was applied to a $\mathrm{Au} / \mathrm{Ti}$ wire with the width of $10 \mu \mathrm{m}$ on a Si chip by an RF generator (33120A, Keysight), The Au/Ti wire was not impedance matched to $50 \Omega$. The direction of the $\mathrm{Au} / \mathrm{Ti}$ wire was set to be parallel to the [110] direction. A static magnetic field along the [111] direction was applied by a pair of $\mathrm{Nd}_{2} \mathrm{Fe}_{14} \mathrm{~B}$ permanent magnets.

The ac magnetic field produced by the $\mathrm{Ti} / \mathrm{Au}$ wire at $15 \mathrm{MHz}$ was simulated by a finitedifference time-domain (FDTD) analysis for comparison with the obtained result. The timedependent Maxwell's equations were solved numerically in the time domain by applying an absorbing boundary condition of a perfectly matched layer ${ }^{23}$ in the total size of the calculated space of $32 \times 60 \times 13 \mathrm{~mm}^{3}$. Variable mesh size was used with the minimum size of the mesh of $0.312 \mu \mathrm{m}$. The total number of the meshes was $268 \times 60 \times 100$ and the time step was 0.52 fs. RF field was fed to the model microstrip structure. The relative permittivities of Si and diamond used in the calculation were 11.20 and 5.68, respectively. 
(a)
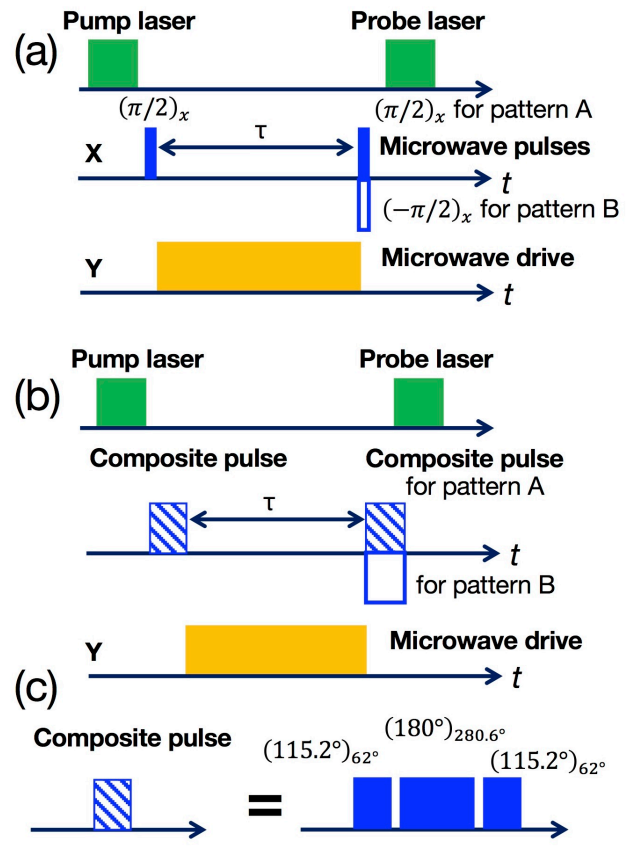

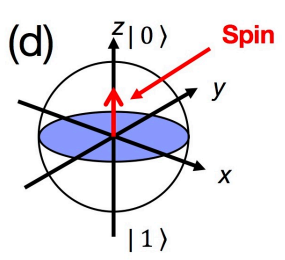

(e)

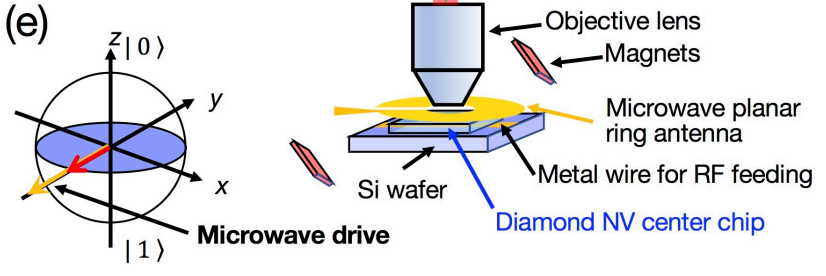

(f) Pattern $A$

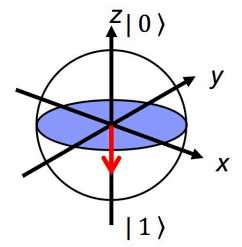

(h)

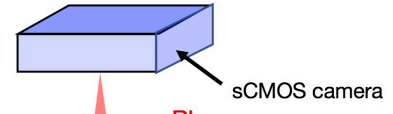

(1) Lens Optical filter Beam splitter

(g) Pattern B

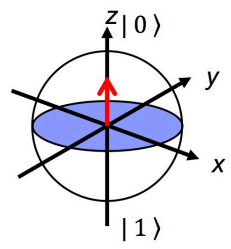

FIG. 1.

Schematics of pulse sequences for spin locking measurement with (a) two $\pi / 2$ pulses and (b) two composite pulses. (c) Schematics of a SCROFULOUS composite pulse composed of three pulses. (d) Evolution of the spin state in the Bloch sphere. The spin state is initialized to the $|0\rangle$ state by the first laser pulse. (e) The first $\pi / 2$ pulse rotates the spin by $90^{\circ}$ to the $(-y)$-direction. A $y$-driving microwave field is applied parallel to the spin in the rotation frame. (f) The second $\pi / 2$ pulse rotates the spin by $90^{\circ}$ to the $(-z)$-direction in the pulse sequence pattern $\mathrm{A}$, or $(\mathrm{g})$ the second $-\pi / 2$ pulse rotates the spin by $-90^{\circ}$ to the $z$-direction in the pulse sequence pattern $\mathrm{B}$. Finally, the spin state is readout from the PL by applying the second laser pulse. (h) Schematics of the experimental setup.

\section{RESULTS AND DISCUSSIONS}

\section{A. $T_{1 \rho}$ relaxation profile}

Figure 2(a) shows a $T_{1 \rho}$ relaxation profile of spin-locking signals using two $\pi / 2$ pulses as shown in Fig. 1(a), fitted with a curve assuming a double exponential profile $A_{1} \exp \left(-t / \tau_{1}\right)+$ $A_{2} \exp \left(-t / \tau_{2}\right)$ with $\tau_{1}=60_{-20}^{+60} \mu \mathrm{s}$, and $\tau_{2}=640_{-200}^{+500} \mu \mathrm{s}$. The initial decay may be due to our measurement method of detecting signals from NV ensembles and depends on extrinsic effects as observed in an NMR measurement ${ }^{24}$ such as the inhomogeneities in microwave field 
or by spin scatterings due to di-vacancies or P1 centers. The long component $T_{1 \rho}=640 \mu \mathrm{s}$ is due to the intrinsic effect, and is significantly longer than both $T_{2}^{*}$ and $T_{2}$ of our diamond chip. This ensures a high sensitivity of the spin-locking measurement.

$T_{1 \rho}$ relaxation profiles were measured by using microwave pulses with the actual Rabi frequency $\Omega^{\prime}$ varied from the target Rabi frequency $\Omega$ as given by $\Omega^{\prime}=(1+g) \Omega$, where $g$ is the fractional error in the microwave pulse amplitude. Figure $2 \mathrm{~b}$ shows that the amplitude error in the microwave pulses does not noticeably change the relaxation profiles with $\tau_{2}=730_{-300}^{+2400}$ $\mu s, 2400_{-1500}^{+\infty} \mu s$, and $3900_{-3000}^{+\infty} \mu s$ for $g=-0.25,-0.375$, and -0.5 , respectively.

\section{B. Noise spectrum}

By varying the amplitude of the driving microwave field, noise spectral function $S_{B}(\Omega)$ is obtained. The rotating-frame relaxation rate $T_{1 \rho}$ is given by $T_{1 \rho}^{-1}=\frac{\gamma_{e}^{2}}{6} S_{B}\left(\Omega_{1}\right)+\frac{1}{2} T_{1}^{-1},{ }^{13,15}$ where $\gamma_{e}$ is the electron gyromagnetic ratio and $T_{1}$ is the spin-lattice relaxation rate, which is $0.86 \mathrm{~ms}$ in our sample measured by phase cycling. Figure 3 shows a noise spectrum acquired by the driving field with the Rabi frequency $\Omega_{\mathrm{d}}$ from 0.27 to $80.4 \mathrm{MHz}$ with a fixed duration of the driving field of $\tau=50 \mu \mathrm{s}$ by the pulse sequence as shown in Fig. 1(a). The spectra in Fig. 3 were obtained by integrating the PL intensity in the area of $1.3 \mu \mathrm{m} \times 13.2 \mu \mathrm{m}$ as indicated in the white square in Fig. 5(a). When the RF field of $-40 \mathrm{dBm}$ was fed to the metal wire structure placed below the diamond chip, a sharp peak at $15 \mathrm{MHz}$ in Fig. 3(b) was detected, which indicates a high frequency- selectivity of the spin-locking measurement. The width of the signal at $15 \mathrm{MHz}$ is mainly given by the inhomogeneous width originated from the variation/fluctuations in the effective Rabi frequency due to variation/fluctuations in the microwave power.

\section{Sensitivity to microwave pulse amplitude error}

In the measurements utilizing wide-field microscopy, the spatial variation in the microwave power in the field of view gives rise to a microwave pulse amplitude error for spin manipulation. In this section, the sensitivity of the detected spin-locking signal on microwave pulse amplitude error is examined. Spin-locking signals are measured by using microwave pulses with the actual Rabi frequency $\Omega^{\prime}=(1+g) \Omega$ varied from the target Rabi frequency 

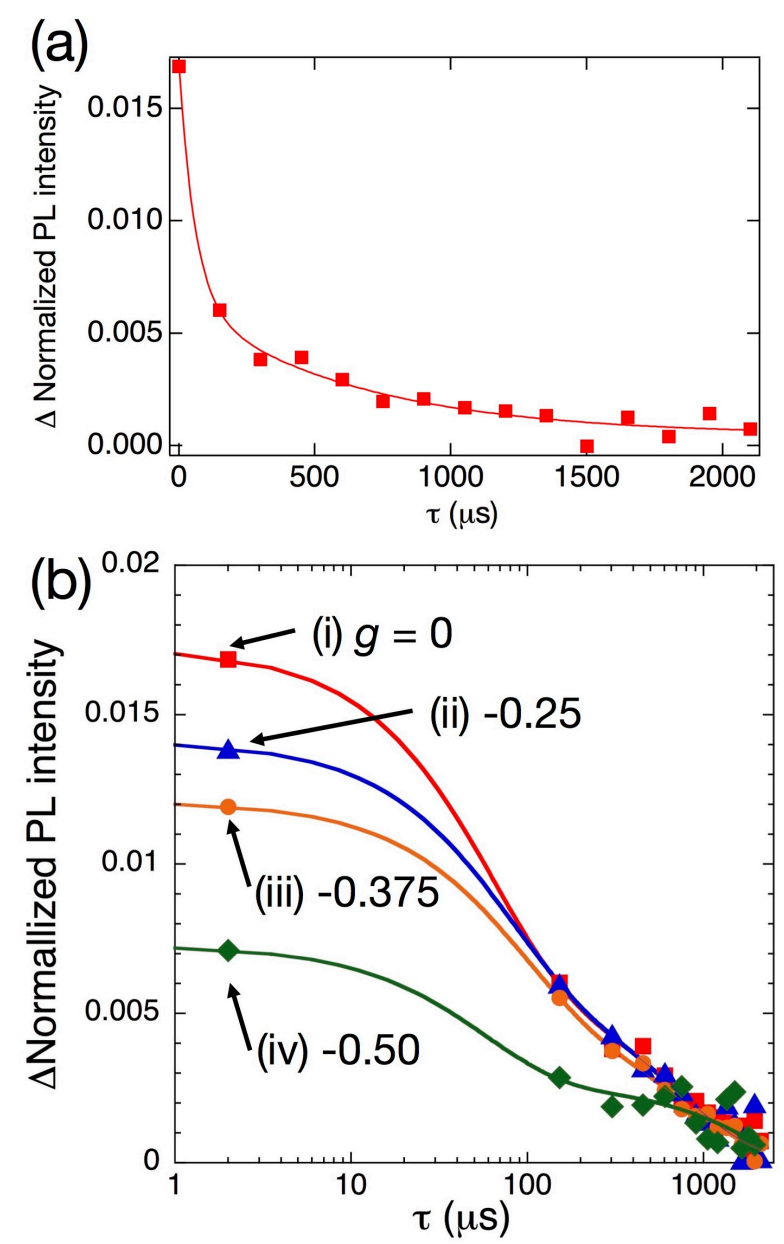

FIG. 2.

(a) $T_{1 \rho}$ relaxation profiles of spin-locking signals in the linear scale using two $\pi / 2$ pulses as shown in Fig. 1(a). (b) Relaxation profiles of spin-locking signals the fractional error in the driving microwave pulse amplitude of (i) 0 , (ii) -0.25 , (iii) -0.375 , (iv) -0.50 in the log scale. The Rabi frequency of the $y$-driving microwave field was set to $\Omega_{d}=26.8 \mathrm{MHz} .\left|I_{A}-I_{B}\right| / I_{B}$ was plotted for clarity.

$\Omega$. Here, the ratio of the microwave intensities of the in-phase and the quadrature-phase components was assumed to be unchanged, i.e., no error was introduced to the phase angle $\phi_{i}$ for SCROFULOUS composite pulse sequence. The Rabi frequency of the $y$-driving microwave field $\Omega_{d}$ was fixed.

Figure 4(a) show the obtained spin locking signals at $g=0$ measured by the sequences using $\pi / 2$ pulses and the SCROFULOUS composite pulses. Spin-locking signal intensity is defined by subtracting the average of the background at points A and B from the peak value 
(a)
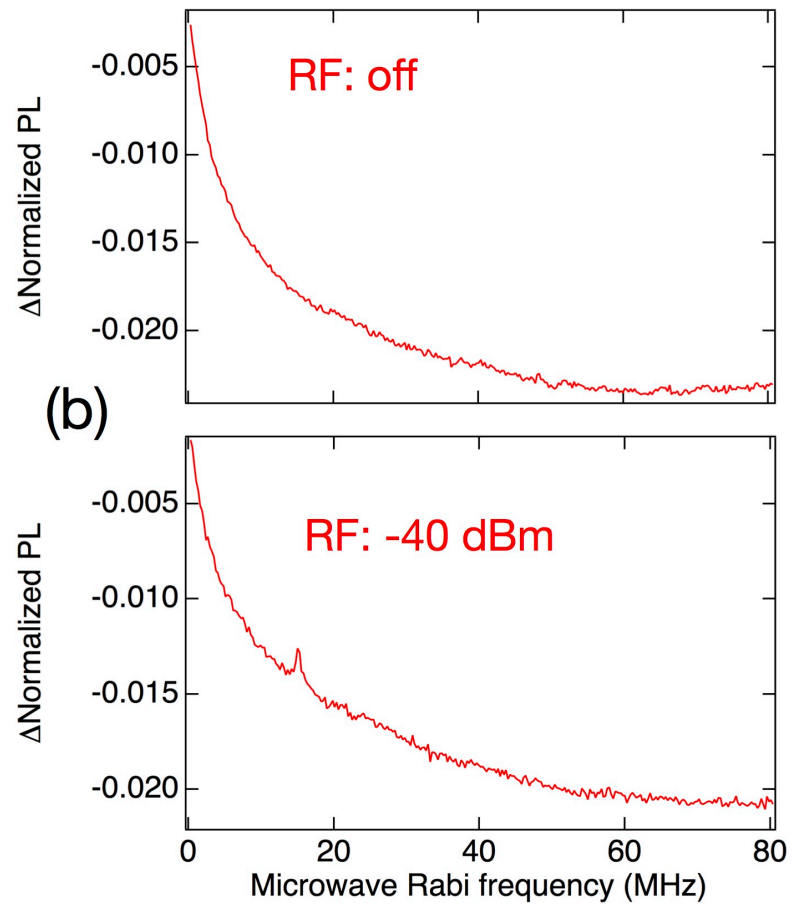

FIG. 3.

Noise spectrum (a) without and (b) with RF field application as a function of the Rabi frequency of the driving field $\Omega$. The duration of the driving field was fixed to $\tau=50 \mu \mathrm{s}$. RF field of -40 $\mathrm{dBm}$ at $15 \mathrm{MHz}$ was applied to the metal wire structure in (b).

at $\mathrm{C}$ as given by $I_{\mathrm{SL}}=I_{\mathrm{PL}}(C)-\left[I_{\mathrm{PL}}(A)+I_{\mathrm{PL}}(B)\right] / 2$, where $I_{\mathrm{PL}}(\mathrm{A}), I_{\mathrm{PL}}(\mathrm{B})$, and $I_{\mathrm{PL}}(\mathrm{C})$ are the normalized PL intensity change $\left(I_{\mathrm{A}}-I_{\mathrm{B}}\right) / I_{\mathrm{B}}$ at the points $\mathrm{A}, \mathrm{B}$, and $\mathrm{C}$, respectively. Points $\mathrm{A}$ and $\mathrm{B}$ were chosen to be the points at which the driving microwave amplitudes were $86 \%$ and $114 \%$ of that at point $\mathrm{C}$.

Figure 4(b) indicates that the SCROFULOUS composite pulses show excellent performance at $|g| \leq 0.25$ and outperforms simple $\pi / 2$ pulses at $-0.625 \leq g \leq 0.5$. At $g=-0.25$, the signal intensity dropped by $19 \%$ in the simple $\pi / 2$ pulse case, whereas in the SCROFULOUS composite pulse case the signal intensity dropped by $2.5 \%$, clearly showing superiority in reducing the sensitivity to pulse amplitude error of SCROFULOUS composite pulses to simple $\pi / 2$ pulses. Whereas Fig. 4(c) indicates that SCROFULOUS composite pulse may be applied in the case of $-0.625 \leq g \leq 0.5$, the performance of the SCROFULOUS composite pulses at $g<-0.625$ and $g>0.5$ was found to be inferior to the performance of the 

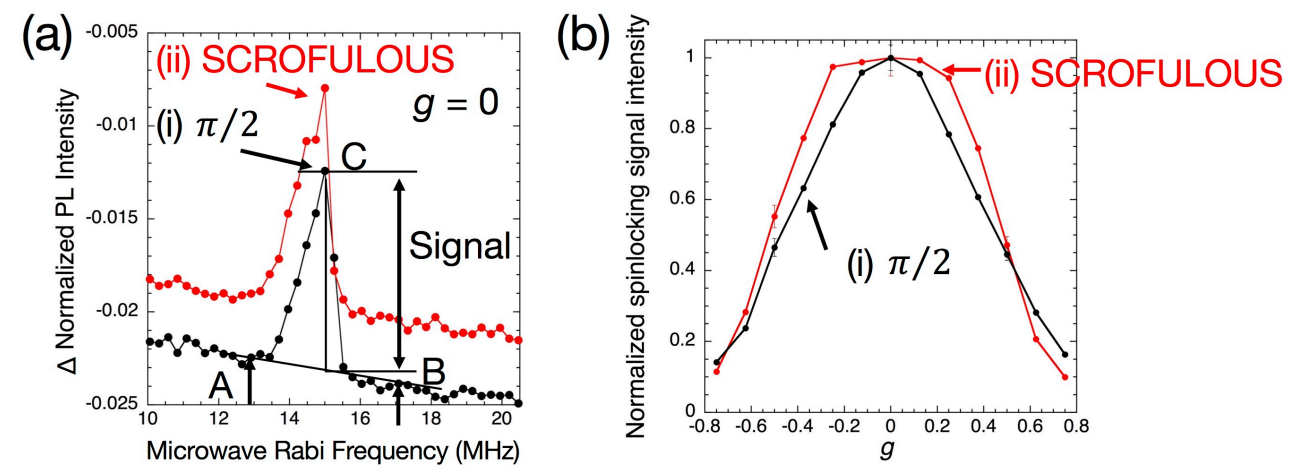

FIG. 4.

Spin locking signals (a) at the fractional error in the driving microwave field $g=0$ for the sequences using (i) $\pi / 2$ pulses and (ii) the SCROFULOUS composite pulses. (b) Spin locking signal as a function of $g$ for the sequences using (i) $\pi / 2$ pulses and (ii) the SCROFULOUS composite pulses. The duration of the driving field was fixed to $\tau=20 \mu \mathrm{s}$. RF field of $-24 \mathrm{dBm}$ at $15 \mathrm{MHz}$ was applied. The duration of a $\pi / 2$ pulse was 9 ns. The error bars were estimated at the representative points of $g=0$ and -0.5 .

simple $\pi / 2$ pulse case. Unlike the theoretical result that showed SCROFULOUS composite pulse outperforms simple $\pi / 2$ pulse in all the range of $g,{ }^{18}$ the relaxation of the spins due to the noise in microwave pulses ${ }^{25}$ restricts the application range of SCROFULOUS composite pulse with a longer total pulse length. The performance of the SCROFULOUS composite pulses is better at $g<0$ than at $g>0$ as shown in Fig. 4(c).

\section{Radio-frequency imaging}

The transition probability under a coherent RF field irradiation at the frequency of $\omega$ is given by ${ }^{14}$

$$
p=p_{0} \frac{\Omega_{1}^{2}}{\Omega_{\mathrm{eff}}^{2}} \sin ^{2}\left(\Omega_{\mathrm{eff}} \tau / 2\right),
$$

where $p_{0}$ is the maximum possible transition probability, $\Omega_{1}$ is the Rabi frequency of the RF field, and $\Omega_{\mathrm{eff}}=\sqrt{\Omega_{1}^{2}+\left(\omega-\Omega_{\mathrm{d}}\right)^{2}}$. The separation of the dressed states is given by the Rabi frequency of the driving microwave field $\Omega_{\mathrm{d}}=\gamma B_{1}^{\mathrm{MW}}$, where $\gamma$ and $B_{1}^{\mathrm{MW}}$ are the gyromagnetic factor, and the amplitude of the driving microwave field, respectively. For a 
weak RF field, the transition probability is approximated by

$$
p=p_{0} \frac{\Omega_{1}^{2} \tau^{2}}{4}
$$

where the Rabi frequency of the RF field $\Omega_{1}$ is directly associated with the amplitude of the RF field $B^{\mathrm{RF}}$ by $\Omega_{1}=\gamma B^{\mathrm{RF}} .{ }^{14}$ Then the spin locking signal proportional to the transition probability $p$ is proportional to the magnitude of the RF field $\left|B^{\mathrm{RF}}\right|^{2}$, which is proportional to the power density of the RF field.

In RF field imaging, the microwave field intensity distribution may affect the accuracy of the result. This is particularly important in measuring RF field distribution around electrically conductive material, where microwave field is locally enhanced by the nearfield. ${ }^{3}$ Two cases are considered here; in the first case, where the measurement target RF frequency is unknown, it is required to measure the microwave field intensity distribution at the frequency for driving NV spins before RF field imaging. This may be performed, for example, by an FFT imaging of Rabi oscillation of the microwave field ${ }^{3}$. Then RF field image is obtained by calibrating the microwave field intensity variation in the field of view. In the second case, where the target RF frequency is fixed and known, an independent measurement of the microwave field distribution may be omitted, instead one may measure the spin-locking signal by applying microwave driving field in the intensity range sufficient to cover the whole range of the microwave field intensity variation in the field of view. This may be applied to the case where an RF field of known frequency is irradiated to a target object, and a re-emitted or scattered RF field image is measured. In the following, we demonstrate RF field imaging of the second case where the target RF frequency is known.

Target Rf field was generated by feeding RF signal of $-30 \mathrm{dBm}$ at $15 \mathrm{MHz}$ to a $\mathrm{Au} / \mathrm{Ti}$ wire with a width of $10 \mu \mathrm{m}$ as shown in Fig. 5(a). A typical spin-locking spectrum is shown in Fig. 5 (b), where the PL was integrated within the area indicated in Fig. 5(a). $\mathrm{RF}$ field projected to the plane perpendicular to the [111] direction of the NV center axis was measured. RF field projected to the planes perpendicular to the other possible three directions of the NV center axis may also be measured by selecting the microwave resonance frequency for the spins in the NV centers.

The amplitude of the driving microwave field was scanned from approximately 10 to 21 $\mathrm{MHz}$ and 41 images were acquired. The maxima of $I_{S L}$ were searched from the acquired images at each pixel and a normalized microwave amplitude image is obtained as shown in 
Fig. 5(c). An RF image is obtained by plotting the spin-locking signal at each pixel as shown in Fig. 5(d). In Figs. 5(c) and 5(d), the images were plotted in the region $10.8<x<30.3$ $\mu \mathrm{m}$ since the image was dominated by noise outside this region. One may obtain the image in the region not shown by adjusting the microwave power of the pulses and the driving field.

The microwave field intensity variation was within $14 \%$ in the region of $10.8<x<30.3$ $\mu \mathrm{m}$ in Fig. 5(c) as estimated from the microwave driving field intensity at the peak position of the spin-locking signal. The variation in the microwave driving field intensity was thus within the range where SCROFULOUS composite pulse shows high fidelity. According to Fig. 4, the detected RF field intensity is estimated to be underestimated by $1.2 \%$ at most. This may be compared with $6.6 \%$ for the case of using simple $\pi / 2$ pulses. In the region $x<10.8 \mu \mathrm{m}$ or $x>30.3 \mu \mathrm{m}$, the spin-locking signal was small, and hence, the peak position of the spin-locking signal was obscured by the noise. The obtained RF field intensity may be calibrated by using the estimated microwave pulse amplitude error.

The spin locking signal as a function of the lateral position as shown in Fig. 6(a) indicate that the RF field projected to the plane perpendicular to the [111] direction has peaks in the vicinity of the edges of the wire. The in-plane magnetic field perpendicular to the direction of the wire is symmetric with respect to the center of the wire and the magnetic field perpendicular to the plane is antisymmetric with respect to the center of the wire. As a result, the magnitude of the detected RF field project to the plane perpendicular to the [111] direction is symmetric with respect to the center of the wire as shown in the calculated result by a finite-difference time-domain (FDTD) method (Fig. 6(b)).

Our method may also be applied in the case of wider microwave field variation in the image area of interest; one may measure spin-locking images by changing the microwave field intensity for spin manipulation, and combine RF field intensity images by selecting the region where $|g|$ is small.

\section{CONCLUSIONS}

We have demonstrated a method to obtain an RF intensity image by utilizing a spinlocking method based on wide-field microscopy of an ensemble of NV centers in diamond. By adopting the SCROFULOUS composite pulse sequence for manipulation of the spins in the 

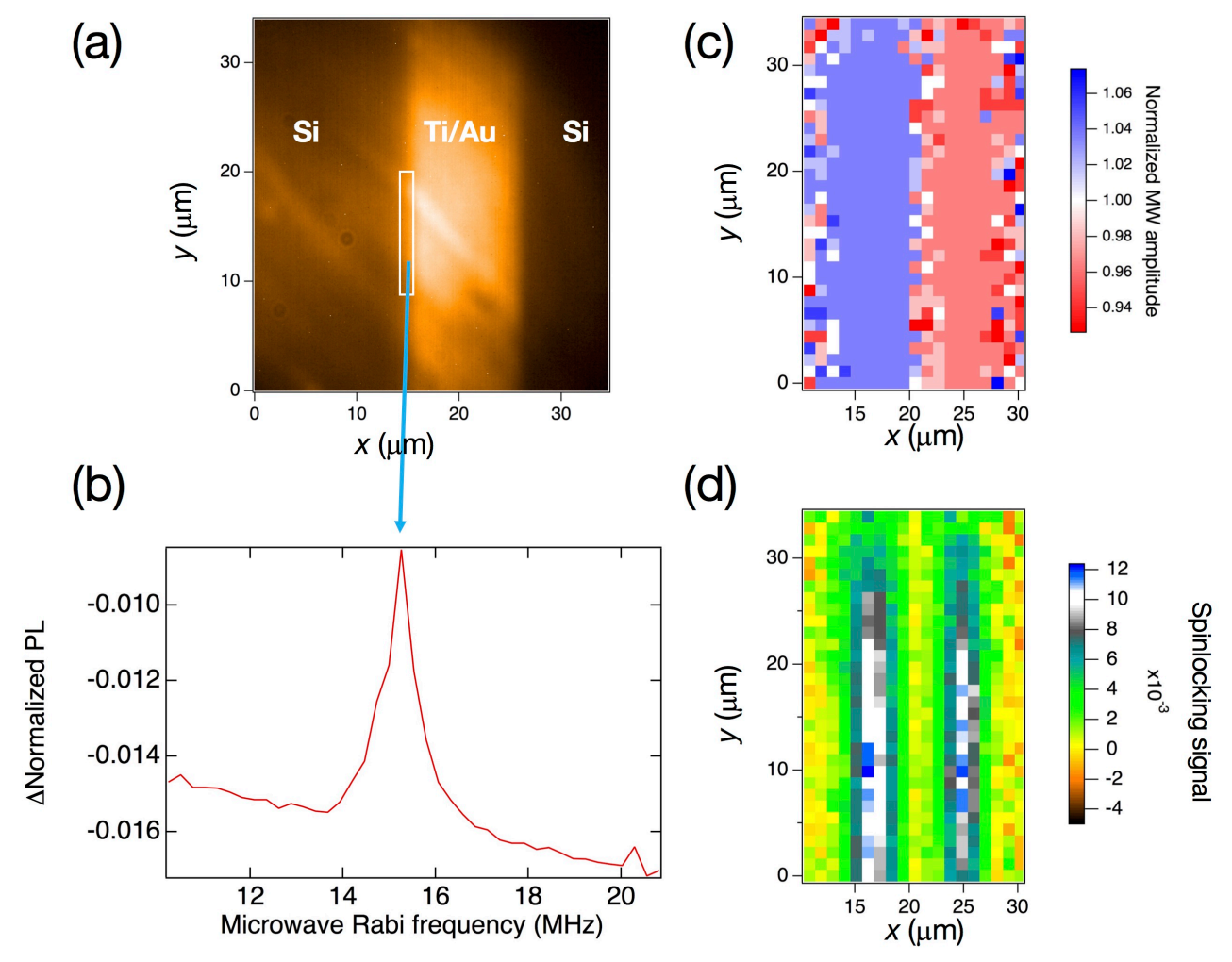

FIG. 5 .

(a) Optical microscope image of a $10 \mu \mathrm{m}$ wide $\mathrm{Ti} / \mathrm{Au}$ wire structure. (b) Spin locking spectrum obtained by integrating the PL in the area shown in the white square in (a). The duration of the driving field was fixed to $\tau=20 \mu \mathrm{s}$. RF field of $-30 \mathrm{dBm}$ was applied. (c) An obtained microwave (MW) amplitude image normalized by 0.547 times the peak MW amplitude of the $\pi / 2$ pulse, corresponding to $\Omega=15 \mathrm{MHz}$ at the center of the Ti/Au wire structure. (d) An obtained RF field image. $528 \times 512$ pixels of the scientific CMOS camera were binned to $33 \times 32$ pixels in (c) and (d).

NV centers, the sensitivity to possible pulse amplitude error is reduced. The spatial resolution of the RF image obtained by our method is markedly higher than the existing methods. The spatial resolution is ultimately limited by the distance of the implanted NV center layer from the surface of the diamond chip of about $10 \mathrm{~nm}$, and is practically limited by the resolution of an optical microscope to be used and may be enhanced by applying super-resolution imaging such as stochastic optical reconstruction microscopy ${ }^{26}$ and stimulated emission depletion microscopy ${ }^{27}$. In our method, the number of pixels of the image is not limited by the number of electric wiring for sensors as used in many existing methods. Furthermore, the 
(a)

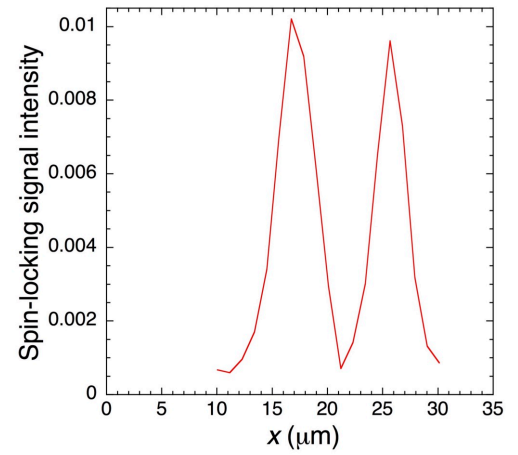

(b)

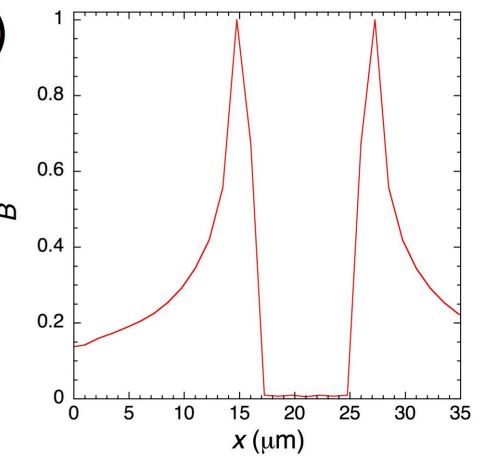

FIG. 6.

Figure 6 (a) Spin locking signal depending as a function of the lateral position. (b) ac magnetic field distribution calculated by an FDTD simulation. The ac magnetic field was normalized to the peak value.

complexity inherent to the mutual inductance of the electric wiring to sensor elements can be avoided since we use NV centers as sensing elements. By applying the spin-locking method, the sensitivity to RF electromagnetic field has been enhanced significantly. Our method may be applied in a broad variety of application areas, for example, material characterizations of such as polar molecules, polymers, and proteins, characterization of components for RF devices such as substrates and RF shields, and medical applications such as early diagnosis of disease and magnetocardiography.

\section{ACKNOWLEDGMENTS}

This work was partly supported by a Grant-in-Aid for Scientific Research (Nos. JP18H04283, JP18H01243, JP18K18726, and JP21H01009) from Japan Society for the Promotion of Science. S.N. acknowledge the stimulating discussion in the meeting of the Cooperative Research Project of the Research Institute of Electrical Communication, Tohoku University.

\section{DATA AVAILABILITY}

The data that support the findings of this study are available from the corresponding author upon reasonable request. 


\section{REFERENCES}

${ }^{1}$ R. Zoughi, Microwave non-destructive testing and evaluation (Kluwer Academic Publishers, Dordrecht, 2000).

${ }^{2}$ S. S. Ahmed, A. Schiessl, F. Gumbmann, M. Tiebout, S. Methfessel, and L.-P. Schmidt, "Advanced microwave imaging," IEEE Microwave Mag. 13, 26-43 (2012).

${ }^{3}$ G. Mariani, S. Nomoto, S. Kashiwaya, and S. Nomura, "System for the remote control and imaging of MW fields for spin manipulation in NV centers in diamond," Sci. Rep. 10, $4813(2020)$.

${ }^{4}$ M. W. Doherty, N. B. Manson, P. Delaney, F. Jelezko, J. Wrachtrup, and L. C. Hollenberg, "The nitrogen-vacancy colour centre in diamond," Phys. Rep. 528, 1 - 45 (2013).

${ }^{5}$ V. Stepanov, F. H. Cho, C. Abeywardana, and S. Takahashi, "High-frequency and highfield optically detected magnetic resonance of nitrogen-vacancy centers in diamond," Appl. Phys. Lett. 106, 063111 (2015).

${ }^{6}$ A. Franchois, A. Joisel, C. Pichot, and J. Bolomey, "Quantitative microwave imaging with a $2.45 \mathrm{GHz}$ planar microwave camera," IEEE Trans. on Med. Imag. 17, 550-561 (1998).

${ }^{7}$ K. Fujiwara, M. Oogane, A. Kanno, M. Imada, J. Jono, T. Terauchi, T. Okuno, Y. Aritomi, M. Morikawa, M. Tsuchida, N. Nakasato, and Y. Ando, "Magnetocardiography and magnetoencephalography measurements at room temperature using tunnel magneto-resistance sensors," Appl. Phys. Exp. 11, 023001 (2018).

${ }^{8}$ Y. Adachi, D. Oyama, Y. Terazono, T. Hayashi, T. Shibuya, and S. Kawabata, "Calibration of room temperature magnetic sensor array for biomagnetic measurement," IEEE TRANS. on Magne. 55, 5000506 (2019).

${ }^{9}$ S. R. Hartmann and E. L. Hahn, "Nuclear double resonance in the rotating frame," Phys. Rev. 128, 2042-2053 (1962).

${ }^{10}$ C. P. Slichter and D. Ailion, "Low-field relaxation and the study of ultraslow atomic motions by magnetic resonance," Phys. Rev. 135, A1099-A1110 (1964).

${ }^{11}$ Y. S. Bai, A. G. Yodh, and T. W. Mossberg, "Selective excitation of dressed atomic states by use of phase-controlled optical fields," Phys. Rev. Lett. 55, 1277-1280 (1985).

${ }^{12}$ H. Ito, T. Ito, and T. Yabuzaki, "Accumulative transfer of transverse magnetic moment between spin-locked Rb and Cs atoms," J. Phys. Soc. Jpn. 63, 1337-1344 (1994). 
${ }^{13}$ F. Yan, S. Gustavsson, J. Bylander, X. Jin, F. Yoshihara, D. G. Cory, Y. Nakamura, T. P. Orlando, and W. D. Oliver, "Rotating-frame relaxation as a noise spectrum analyser of a superconducting qubit undergoing driven evolution," Nature Comm. 4 (2013).

${ }^{14}$ M. Loretz, T. Rosskopf, and C. L. Degen, "Radio-frequency magnetometry using a single electron spin," Phys. Rev. Lett. 110, 017602 (2013).

${ }^{15}$ T. Rosskopf, A. Dussaux, K. Ohashi, M. Loretz, R. Schirhagl, H. Watanabe, S. Shikata, K. M. Itoh, and C. L. Degen, "Investigation of surface magnetic noise by shallow spins in diamond," Phys. Rev. Lett. 112, 147602 (2014).

${ }^{16}$ M. H. Levitt, "Composite pulses," Prog. Nucl. Magn. Reson. Spectrosc. 18, 61-122 (1986).

${ }^{17} \mathrm{~S}$. Wimperis, "Broadband, narrowband, and passband composite pulses for use in advanced NMR experiments," J. Magn. Reson., Ser. A. 109, 221-231 (1994).

${ }^{18}$ H. K. Cummins, G. Llewellyn, and J. A. Jones, "Tackling systematic errors in quantum logic gates with composite rotations," Phys. Rev. A 67, 042308 (2003).

${ }^{19}$ J. A. Jones, "Robust ising gates for practical quantum computation," Phys. Rev. A 67, $012317(2003)$.

${ }^{20}$ J. Zopes, K. Sasaki, K. S. Cujia, J. M. Boss, K. Chang, T. F. Segawa, K. M. Itoh, and C. L. Degen, "High-resolution quantum sensing with shaped control pulses," Phys. Rev. Lett. 119, 260501 (2017).

${ }^{21}$ K. Sasaki, Y. Monnai, S. Saijo, R. Fujita, H. Watanabe, J. Ishi-Hayase, K. M. Itoh, and E. Abe, "Broadband, large-area microwave antenna for optically detected magnetic resonance of nitrogen-vacancy centers in diamond," Rev. Sci. Instrum. 87, 053904 (2016). ${ }^{22}$ B. K. Ofori-Okai, S. Pezzagna, K. Chang, M. Loretz, R. Schirhagl, Y. Tao, B. A. Moores, K. Groot-Berning, J. Meijer, and C. L. Degen, "Spin properties of very shallow nitrogen vacancy defects in diamond," Phys. Rev. B 86, 081406 (2012).

${ }^{23}$ A. Oga, "How to use OpenFDTD (in japanese)," RF World 39, 56-72 (2017).

${ }^{24}$ S. Sasaki, T. Miura, K. Ikeda, M. Sakai, T. Sekikawa, M. Saito, T. Yuge, and Y. Hirayama, " $1 / f^{2}$ spectra of decoherence noise on ${ }^{75}$ As nuclear spins in bulk GaAs," Sci. Rep. 10, 10674 (2020).

${ }^{25}$ J.-M. Cai, B. Naydenov, R. Pfeiffer, L. P. McGuinness, K. D. Jahnke, F. Jelezko, M. B. Plenio, and A. Retzker, "Robust dynamical decoupling with concatenated continuous driving," New J. Phys. 14, 113023 (2012). 
${ }^{26}$ M. Pfender, N. Aslam, G. Waldherr, P. Neumann, and J. Wrachtrup, "Single-spin stochastic optical reconstruction microscopy," Proc. Natl. Acad. Sci. 111, 14669-14674 (2014).

${ }^{27}$ Y. Silani, F. Hubert, and V. M. Acosta, "Stimulated emission depletion microscopy with diamond silicon vacancy centers," ACS Photonics 6, 2577-2582 (2019). 